\title{
New stellar $(n, \gamma)$ cross sections and the "Karlsruhe Astrophysical Database of Nucleosynthesis in Stars"
}

\author{
I. Dillmann ${ }^{1,2, a}$, R. Plag ${ }^{1,3}$, C. Domingo-Pardo ${ }^{1}$, M. Heil ${ }^{1,3}$, F. Käppeler ${ }^{1}$, T. Rauscher ${ }^{2}$, and F.-K. Thielemann ${ }^{2}$ \\ ${ }^{1}$ Institut für Kernphysik, Forschungszentrum Karlsruhe, Postfach 3640, 76021 Karlsruhe, Germany \\ 2 Departement Physik und Astronomie, Universität Basel, Klingelbergstrasse 82, 4056 Basel, Switzerland \\ 3 Gesellschaft für Schwerionenforschung mbH, Plankstrasse 1, 64291 Darmstadt, Germany
}

\begin{abstract}
Since April 2005 a regularly updated stellar neutron cross section compilation is available online at http://nuclear-astrophysics.fzk.de/kadonis. This online-database is called the "Karlsruhe Astrophysical Database of Nucleosynthesis in Stars" project and is based on the previous Bao et al. compilation from the year 2000. The present version KADoNiS v0.2 (January 2007) includes recommended cross sections for 280 isotopes between ${ }^{1} \mathrm{H}$ and ${ }^{210} \mathrm{Po}$ and 75 semi-empirical estimates for isotopes without experimental information. Concerning stellar $(n, \gamma)$ cross sections of the 32 stable, proton-rich isotopes produced by the $p$ process experimental information is only available for 20 isotopes, but 9 of them have rather large uncertainties of $\geq 9 \%$. The first part of a systematic study of stellar $(n, \gamma)$ cross sections of the $p$-process isotope ${ }^{74} \mathrm{Se},{ }^{84} \mathrm{Sr},{ }^{102} \mathrm{Pd},{ }^{120} \mathrm{Te},{ }^{130} \mathrm{Ba},{ }^{132} \mathrm{Ba},{ }^{156} \mathrm{Dy}$, and ${ }^{174} \mathrm{Hf}$ is presented. In another application KADoNiS v0.2 was used for an modification of a reaction library of Basel university. With this modified library $p$-process network calculations were carried out and compared to previous results.
\end{abstract}

\section{Stellar neutron capture compilations}

The pioneering work for stellar neutron capture cross sections was published in 1971 by Allen and co-workers [1]. In this paper the role of neutron capture reactions in the nucleosynthesis of heavy elements was reviewed and a list of recommended (experimental or semi-empirical) Maxwellian averaged cross sections at $k T=30 \mathrm{keV}$ (MACS30) presented for nuclei between $\mathrm{C}$ and $\mathrm{Pu}$.

The idea of an experimental and theoretical stellar neutron cross section database was picked up again by Bao and Käppeler [2] for $s$-process studies. This compilation published in 1987 included cross sections for $(n, \gamma)$ reactions (between ${ }^{12} \mathrm{C}$ and ${ }^{209} \mathrm{Bi}$ ), some $(n, p)$ and $(n, \alpha)$ reactions (for ${ }^{33} \mathrm{Se}$ to $\left.{ }^{59} \mathrm{Ni}\right)$, and also $(n, \gamma)$ and $(n, f)$ reactions for long-lived actinides. A follow-up compilation was published by Beer et al. in 1992 [3].

In the update of 2000 the Bao compilation [4] was extended down to ${ }^{1} \mathrm{H}$ and - like the original Allen paper semi-empirical recommended values for nuclides without experimental cross section information were added. These estimated values are normalized cross sections derived with the Hauser-Feshbach code NON-SMOKER [5], which account for known systematic deficiencies in the nuclear input of the calculation. Additionally, the database provided stellar enhancement factors and energy-dependent MACS for energies between $k T=5 \mathrm{keV}$ and $100 \mathrm{keV}$.

The KADoNiS project [6] is based on these previous compilations and aims to be a regularly updated database. The current version KADoNiS v0.2 (January 2007) is already the second update and includes - compared to the previous Bao et al. compilation [4] - 38 updated and 14 new recommended cross sections. The update history can be followed in the section "Logbook". A paper version of KADoNiS ("v1.0")

\footnotetext{
${ }^{a}$ Presenting author, e-mail: iris.dillmann@ik.fzk.de
}

is planned for 2008, which also will - like the first Bao compilation from 1987 [2] - include $(n, p)$ and $(n, \alpha)$ reactions for light isotopes and $(n, \gamma)$ and $(n, f)$ reactions for longlived actinides at $k T=30 \mathrm{keV}$. Additionally, a re-calculation of semi-empirical estimates based on the latest experimental results of neighboring nuclides will be performed.

\section{Systematic study of $(n, \gamma)$ cross sections for the $p$ process}

\subsection{The " $p$ processes"}

The " $p$ process" is responsible for the production of 32 stable but rare isotopes between ${ }^{74} \mathrm{Se}$ and ${ }^{196} \mathrm{Hg}$ on the proton-rich side of the valley of stability. Unlike the remaining $99 \%$ of the heavy nuclei beyond iron these isotopes cannot be created by neutron captures in the $s$ process or $r$ process, and their solar [7] and isotopic abundances [8] are 1-2 orders of magnitude lower than the respective $s$ - and $r$-process nuclei. The bulk of $p$ isotopes is thought to be produced in explosive $\mathrm{O} / \mathrm{Ne}$ burning during supernova type II explosions (core collapse supernovae). This mechanism is called " $\gamma$ process" since the main reactions are photo-induced reactions of high energy photons $\left(T_{9}=2-3\right)$ on pre-existing seed nuclei from prior $s$-processing. The " $\gamma$ process" can reproduce the solar abundances [7] of most $p$ isotopes within a factor of $3[9,10]$.

For the missing abundances of the most abundant isotopes ${ }^{92,94} \mathrm{Mo}$ and ${ }^{96,98} \mathrm{Ru}$ alternative processes have been proposed, e.g. using strong neutrino fluxes in the " $v p$ process" [11], or rapid proton-captures in the " $r p$ process" [12] in a binary, cataclysmic system with a neutron star accreting material from a Red Giant.

Apart from the astrophysical uncertainties of the $p$-process site another problem arises from the large nuclear physics 
uncertainties due to missing experimental cross section data. Present network calculations for the reproduction of the solar abundances of the $32 p$-process isotopes include up to now only the $\gamma$-process scenario since it seems to be the best understood part of the $p$ processes. Such network calculations are carried out with a typical reaction library consisting of $\approx 1600$ isotopes which are connected by several thousands of reactions $[13,14]$. The largest fraction of these reactions concerns short-lived radioactive nuclei and thus have to be inferred from theoretical work, e.g. the Hauser-Feshbach statistical model $[5,15]$.

Some experimental information for the $p$ process is available for charged-particle reactions, but the largest amount of data concerns $(n, \gamma)$ data, which is connected via detailed balance with the respective $(\gamma, n)$ reactions needed for the $\gamma$ process. However, most of this neutron capture data was measured with the activation technique at one single energy $(k T=25 \mathrm{keV})$, and thus has to be extrapolated to the respective $\gamma$-process energies $(k T=170-260 \mathrm{keV})$ with the help of energy-dependencies from Hauser-Feshbach theory. This method was used in the modification of a reaction library discussed in section 3 .

\subsection{Experimental technique}

The previous status of $(n, \gamma)$ cross sections for the $32 p$ isotopes at $k T=30 \mathrm{keV}$ is listed in the third column of table 3 [4]. Experimental data was available for 20 isotopes but 9 of them $\left({ }^{92,94} \mathrm{Mo},{ }^{96} \mathrm{Ru},{ }^{124,126} \mathrm{Xe},{ }^{130} \mathrm{Ba},{ }^{156} \mathrm{Dy},{ }^{180} \mathrm{~W}\right.$, and $\left.{ }^{190} \mathrm{Pt}\right)$ exhibited uncertainties larger than $9 \%$. For the remaining 12 isotopes no experimental information was available in the stellar energy range, where only semi-empirical estimates based on Hauser-Feshbach predictions existed.

This motivated an extended measuring campaign at the Karlsruhe 3.7 MV Van de Graaff accelerator using the activation technique. In this framework the $p$ isotopes ${ }^{74} \mathrm{Se},{ }^{84} \mathrm{Sr}$, ${ }^{102} \mathrm{Pd},{ }^{120} \mathrm{Te},{ }^{130} \mathrm{Ba},{ }^{132} \mathrm{Ba},{ }^{156} \mathrm{Dy}$, and ${ }^{174} \mathrm{Hf}$ were measured with samples of natural composition. Neutrons were produced via the ${ }^{7} \mathrm{Li}(p, n){ }^{7} \mathrm{Be}$ reaction by bombarding $10-30 \mu \mathrm{m}$ thick layers of metallic lithium or lithiumfluoride on a water-cooled copper backing with protons of $\mathrm{E}_{p}=1912 \mathrm{keV}, 31 \mathrm{keV}$ above the ${ }^{7} \mathrm{Li}(p, n)$ reaction threshold at $1881 \mathrm{keV}$. The resulting quasi-stellar neutron spectrum approximates a Maxwellian distribution for $k T=25.0 \pm 0.5 \mathrm{keV}$ [16] but is truncated at $E_{n}=106 \mathrm{keV}$. Under these conditions, all neutrons are kinematically collimated into a forward cone of $120^{\circ}$ opening angle. Neutron scattering through the $\mathrm{Cu}$ backing is negligible, since the transmission is $\approx 98 \%$ in the energy range of interest.

The sample materials were either metals ( $\mathrm{Se}, \mathrm{Pd}, \mathrm{Te}, \mathrm{Dy}$, and $\mathrm{Hf}$ ) or compounds ( $\left.\mathrm{SrO}, \mathrm{SrCO}_{3}, \mathrm{SrF}_{2}, \mathrm{BaCO}_{3}\right)$. Thin pellets were pressed from the respective powders or granules and enclosed in cans made from thin aluminium foil. In case of $\mathrm{Pd}, \mathrm{Dy}$, and Hf the samples were cut from thin metal foils. During the activations the samples were sandwiched between $10-30 \mu \mathrm{m}$ thick gold foils of the same diameter and were irradiated in close geometry to the neutron target. In this way the neutron flux can be determined relative to the well-known capture cross section of ${ }^{197} \mathrm{Au}$ [16]. The activation measurements were carried out with the Van de
Graaff accelerator operated in DC mode with a current of $\approx 100 \mu \mathrm{A}$ (for the Li targets) or even higher currents (up to $150 \mu \mathrm{A}$ ) for the $\mathrm{LiF}$ targets. The mean neutron flux over the period of the activations was $\approx 1.5-3 \times 10^{9} \mathrm{~s}^{-1}$ at the position of the samples. To ensure homogeneous illumination of the entire surface the proton beam was wobbled across the $\mathrm{Li}$ target. During the irradiation the neutron flux was recorded in intervals of $60 \mathrm{~s}$ or $90 \mathrm{~s}$ using a ${ }^{6} \mathrm{Li}$-glass detector for later correction of the number of nuclei which decayed during the activation.

For the measurement of the induced activities two detector setups were available. A single high purity Germanium (HPGe) detector with a well defined geometry and $10 \mathrm{~cm}$ lead shielding was used in all cases for the counting of the gold foils, as well as for the activities of ${ }^{75} \mathrm{Se},{ }^{85} \mathrm{Sr},{ }^{121} \mathrm{Te},{ }^{131} \mathrm{Ba}$, ${ }^{133 m} \mathrm{Ba},{ }^{157} \mathrm{Dy}$, and ${ }^{175} \mathrm{Hf}$. The activities of ${ }^{103} \mathrm{Pd}$ and ${ }^{133 g} \mathrm{Ba}$ were measured with a gamma detection system consisting of two HPGe Clover detectors [17] in close geometry. The decay properties of the determined product nuclei are given in table 1 . The sample and activation parameters are shortly summarized in table 2 .

Table 1. Decay properties of the product nuclei. Shown here are only the strongest transitions used for the analysis.

\begin{tabular}{|c|c|c|c|c|}
\hline Isotope & $t_{1 / 2}$ & $\mathrm{E}_{\gamma}[\mathrm{keV}]$ & $\mathrm{I}_{\gamma}[\%]$ & Ref. \\
\hline \multirow{2}{*}{${ }^{75} \mathrm{Se}$} & 119.79 (4) d & 136.0 & $58.3(7)$ & [18] \\
\hline & & 264.7 & $58.9(3)$ & \\
\hline${ }^{85} \mathrm{Sr}^{\mathrm{g}}$ & $64.84(2) d$ & 514.0 & $95.7(40)$ & [19] \\
\hline \multirow[t]{2}{*}{${ }^{85} \mathrm{Sr}^{\mathrm{m}}$} & $67.63(4) \mathrm{min}$ & 151.2 & $12.9(7)$ & \\
\hline & & 231.9 & $84.4(22)$ & \\
\hline${ }^{103} \mathrm{Pd}$ & $16.991(19) \mathrm{d}$ & 357.5 & $2.21 \times 10^{-2}(7)$ & [20] \\
\hline${ }^{121} \mathrm{Te}^{\mathrm{g}}$ & $19.16(5) \mathrm{d}$ & 573.1 & $80.3(25)$ & [21] \\
\hline \multirow[t]{2}{*}{${ }^{121} \mathrm{Te}^{\mathrm{m}}$} & $154(7) d$ & 212.2 & $81.4(1)$ & \\
\hline & & 1102.1 & $2.54(6)$ & \\
\hline \multirow[t]{4}{*}{${ }^{131} \mathrm{Ba}^{\mathrm{g}}$} & $11.50(6) \mathrm{d}$ & 123.8 & $29.0(3)$ & [22] \\
\hline & & 216.1 & $19.7(2)$ & \\
\hline & & 373.2 & $14.0(2)$ & \\
\hline & & 496.3 & $46.8(2)$ & \\
\hline${ }^{133} \mathrm{Ba}^{\mathrm{g}}$ & $10.52(13) \mathrm{yr}$ & 356.0 & $62.1(2)$ & [23] \\
\hline${ }^{133} \mathrm{Ba}^{\mathrm{m}}$ & 38.9 (1) h & 275.9 & $17.8(6)$ & [24] \\
\hline${ }^{157}$ Dy & $8.14(4) \mathrm{h}$ & 326.3 & $92(4)$ & [25] \\
\hline${ }^{175} \mathrm{Hf}$ & $70(2) d$ & 343.4 & $84.0(30)$ & [26] \\
\hline${ }^{198} \mathrm{Au}$ & $2.69517(21) d$ & 411.8 & $95.58(12)$ & [27] \\
\hline
\end{tabular}

\subsection{Results}

For a detailed description of the data analysis and the results, see $[14,28,29]$. The resulting Maxwellian averaged cross sections at $k T=30 \mathrm{keV}$ from this measuring campaign are shown in Table 3 in bold. The semi-empirical estimates for ${ }^{74} \mathrm{Se},{ }^{102} \mathrm{Pd},{ }^{120} \mathrm{Te},{ }^{132} \mathrm{Ba}$, and ${ }^{174} \mathrm{Hf}$ are reproduced within the large error bars of the prediction. The previous experimental values for ${ }^{130} \mathrm{Ba}$ and ${ }^{156} \mathrm{Dy}$ are confirmed perfectly but with much improved uncertainties. 
Table 2. Activation schemes and sample characteristics. $\Phi_{t o t}$ is the total neutron exposure of the sample during the activation.

\begin{tabular}{cccc}
\hline Isotope & $\begin{array}{c}\text { N(Isotope) } \\
\text { [atoms] }\end{array}$ & $\begin{array}{c}\mathrm{t}_{\text {cct }} \\
\text { [min] }\end{array}$ & $\begin{array}{c}\Phi_{\text {tot }} \\
\text { [neutrons] }\end{array}$ \\
\hline${ }^{74} \mathrm{Se}$ & $(0.7-1.4) \times 10^{19}$ & $419-1425$ & $(0.2-1.5) \times 10^{14}$ \\
${ }^{84} \mathrm{Sr} \rightarrow{ }^{\mathrm{g}}$ & $(0.2-1.3) \times 10^{19}$ & $1234-2621$ & $(0.8-1.6) \times 10^{14}$ \\
${ }^{84} \mathrm{Sr} \rightarrow{ }^{\mathrm{m}}$ & $(0.4-0.8) \times 10^{19}$ & $155-274$ & $(1.1-3.3) \times 10^{13}$ \\
${ }^{102} \mathrm{Pd}$ & $(1.7-2.6) \times 10^{19}$ & $5751-9770$ & $(3.5-8.2) \times 10^{14}$ \\
${ }^{120} \mathrm{Te} \rightarrow \mathrm{g}, \mathrm{m}$ & $(1.6-2.0) \times 10^{18}$ & $1406-4142$ & $(1.5-3.1) \times 10^{14}$ \\
${ }^{130} \mathrm{Ba}$ & $(3.5-4.8) \times 10^{17}$ & $4014-7721$ & $(2.7-6.9) \times 10^{14}$ \\
${ }^{132} \mathrm{Ba} \rightarrow \mathrm{g}, \mathrm{m}$ & $(3.3-4.7) \times 10^{17}$ & $4014-7721$ & $(2.7-6.9) \times 10^{14}$ \\
${ }^{156} \mathrm{Dy}$ & $(0.6-1.8) \times 10^{17}$ & $362-964$ & $(4.0-9.5) \times 10^{13}$ \\
${ }^{174} \mathrm{Hf}$ & $(5.0-8.8) \times 10^{17}$ & $3865-5451$ & $(5.1-8.7) \times 10^{14}$ \\
\hline
\end{tabular}

Thus, only $6 p$ isotopes $\left({ }^{98} \mathrm{Ru},{ }^{138} \mathrm{La},{ }^{158} \mathrm{Dy},{ }^{168} \mathrm{Yb},{ }^{184} \mathrm{Os}\right.$, and ${ }^{196} \mathrm{Hg}$ ) remain without any experimental information about the stellar neutron cross section. The present work is therefore being extended to the heavier $p$ isotopes and includes the measurement of ${ }^{158} \mathrm{Dy},{ }^{168} \mathrm{Yb},{ }^{184} \mathrm{Os}$, and ${ }^{196} \mathrm{Hg}$, and the remeasurement of ${ }^{180} \mathrm{~W}$ and ${ }^{190} \mathrm{Pt}$. Only for ${ }^{98} \mathrm{Ru}$ and ${ }^{138} \mathrm{La}$ the activation technique cannot be applied.

\section{3 p-process simulations with an updated reaction library}

The $p$-process network calculations in $[13,14]$ were carried out with the program "PProSim" $[13,30]$. The underlying network was originally based on a reaction library from Michigan State University for X-ray bursts, which included only proton-rich isotopes up to Xenon. For $p$-process studies it was extended with a full reaction network library from Basel university [31]. This reaction library is mainly based on NONSMOKER predictions [5] with only a few experimental information for light nuclei, and was modified with the latest stellar neutron capture cross sections available from KADoNiS v0.2. This modification includes more than 350 experimental and semi-empirical $(n, \gamma)$ cross sections and was extended to the respective $(\gamma, n)$ channels calculated via detailed balance.

PProSim simulates the abundance evolution for the $32 p$ isotopes with a parameterized model of a supernova type II explosion of a $25 \mathrm{M}_{\odot}$ star. Since the $p$-process layers are located far outside the collapsing core, they only experience the bounced shock front passing through the O/Ne burning zone and the subsequent temperature and density increase. Both, the seed abundances and the respective temperature and density profiles, were taken from external works and not calculated self-consistently (for more information, see [13]).

The results from the simulations with the modified reaction library were compared to the results published in [13] to examine the influence of the experimental neutron capture data. This was done with help of the so-called "normalized overproduction factor", which is $=1$ when the calculated abundance corresponds to the solar abundances [7]. Ranges of variations of this factor for SN type II explosions with star
Table 3. Status of MACS30 of all $32 p$ nuclei. Isotopic abundances were taken from [8]. Recommended cross section were taken from [4] and [6]. Italic values show semi-empirical estimates. *Preliminary semi-empirical value based on KADoNiS v0.2. **Preliminary value, data analysis not yet fully completed.

\begin{tabular}{|c|c|c|c|}
\hline \multirow[t]{3}{*}{ Isotope } & \multirow{3}{*}{$\begin{array}{c}\text { Isotopic } \\
\text { abundance [8] } \\
{[\%]}\end{array}$} & \multicolumn{2}{|c|}{ Recommended MACS30 } \\
\hline & & previous [4] & new [6] \\
\hline & & {$[\mathrm{mb}]$} & {$[\mathrm{mb}]$} \\
\hline${ }^{74} \mathrm{Se}$ & $0.89(4)$ & $267 \pm 25$ & $271 \pm 15[28]$ \\
\hline${ }^{78} \mathrm{Kr}$ & $0.35(1)$ & \multicolumn{2}{|c|}{$312 \pm 26$} \\
\hline${ }^{84} \mathrm{Sr}$ & $0.56(1)$ & $368 \pm 125$ & $\mathbf{3 0 0} \pm \mathbf{1 7}[28]$ \\
\hline${ }^{92} \mathrm{Mo}$ & $14.84(35)$ & \multicolumn{2}{|c|}{$70 \pm 10$} \\
\hline${ }^{94} \mathrm{Mo}$ & $9.25(12)$ & \multicolumn{2}{|c|}{$102 \pm 20$} \\
\hline${ }^{96} \mathrm{Ru}$ & $5.54(14)$ & $238 \pm 60$ & $207 \pm 8$ \\
\hline${ }^{98} \mathrm{Ru}$ & $1.87(3)$ & \multicolumn{2}{|c|}{$173 \pm 36$} \\
\hline${ }^{102} \mathrm{Pd}$ & $1.02(1)$ & $373 \pm 118$ & $370 \pm 14$ \\
\hline${ }^{106} \mathrm{Cd}$ & $1.25(6)$ & \multicolumn{2}{|c|}{$302 \pm 24$} \\
\hline${ }^{108} \mathrm{Cd}$ & $0.89(3)$ & \multicolumn{2}{|c|}{$202 \pm 9$} \\
\hline${ }^{113} \mathrm{In}$ & $4.29(5)$ & \multicolumn{2}{|c|}{$787 \pm 70$} \\
\hline${ }^{112} \mathrm{Sn}$ & $0.97(1)$ & \multicolumn{2}{|c|}{$210 \pm 12$} \\
\hline${ }^{114} \mathrm{Sn}$ & $0.66(1)$ & \multicolumn{2}{|c|}{$134.4 \pm 1.8$} \\
\hline${ }^{115} \mathrm{Sn}$ & $0.34(1)$ & \multicolumn{2}{|c|}{$342.4 \pm 8.7$} \\
\hline${ }^{120} \mathrm{Te}$ & $0.09(1)$ & $420 \pm 103$ & $499 \pm 24$ \\
\hline${ }^{124} \mathrm{Xe}$ & 0.09 (1) & \multicolumn{2}{|c|}{$644 \pm 83$} \\
\hline${ }^{126} \mathrm{Xe}$ & $0.09(1)$ & \multicolumn{2}{|c|}{$359 \pm 51$} \\
\hline${ }^{130} \mathrm{Ba}$ & $0.106(1)$ & $760 \pm 110$ & $767 \pm 30$ \\
\hline${ }^{132} \mathrm{Ba}$ & $0.101(1)$ & $379 \pm 137$ & $399 \pm 16$ \\
\hline${ }^{136} \mathrm{Ce}$ & $0.185(2)$ & \multicolumn{2}{|c|}{$328 \pm 21$} \\
\hline${ }^{138} \mathrm{Ce}$ & $0.251(2)$ & \multicolumn{2}{|c|}{$179 \pm 5$} \\
\hline${ }^{138} \mathrm{La}$ & 0.090 (1) & \multicolumn{2}{|c|}{$419 \pm 59 *$} \\
\hline${ }^{144} \mathrm{Sm}$ & $3.07(7)$ & \multicolumn{2}{|c|}{$92 \pm 6$} \\
\hline${ }^{156}$ Dy & $0.06(1)$ & $1567 \pm 145$ & $1548 \pm 30 * *$ \\
\hline${ }^{158}$ Dy & $0.10(1)$ & \multicolumn{2}{|c|}{$1060 \pm 400$} \\
\hline${ }^{162} \mathrm{Er}$ & $0.14(1)$ & \multicolumn{2}{|c|}{$1624 \pm 124$} \\
\hline${ }^{168} \mathrm{Yb}$ & $0.13(1)$ & \multicolumn{2}{|c|}{$1160 \pm 400$} \\
\hline${ }^{174} \mathrm{Hf}$ & $0.16(1)$ & $956 \pm 283^{*}$ & $983 \pm 46[29]$ \\
\hline${ }^{180} \mathrm{~W}$ & $0.12(1)$ & \multicolumn{2}{|c|}{$536 \pm 60$} \\
\hline${ }^{184} \mathrm{Os}$ & $0.02(1)$ & \multicolumn{2}{|c|}{$657 \pm 202$} \\
\hline${ }^{190} \mathrm{Pt}$ & $0.014(1)$ & \multicolumn{2}{|c|}{$677 \pm 82$} \\
\hline${ }^{196} \mathrm{Hg}$ & $0.15(1)$ & \multicolumn{2}{|c|}{$650 \pm 82$} \\
\hline
\end{tabular}

masses $13 M_{\odot} \leq M_{\star} \leq 25 M_{\odot}$ are published e.g. in figure 4 in [10].

The new overproduction factors are slightly below previously published values $[10,13]$ due to the inclusion of recent experimental data, especially in the mass range $150 \leq A \leq 170$ where the main reaction flux is driven by $(\gamma, n),(\gamma, \alpha),(n, \gamma)$, and $(n, \alpha)$ reactions. All of these reaction fluxes are found to be smaller. Our study underlines the importance of $(n, \gamma)$ and $(\gamma, n)$ reactions in the $p$-process flow. For example, we were able to show that a variation in the neutron rates of the $\mathrm{Pb}$ and $\mathrm{Bi}$ isotopes has a strong impact on the above mentioned fluxes. This is due to the fact that a significant 
fraction of the seed abundances is located in these isotopes and converted to nuclei at lower mass by photodisintegration sequences starting with $(\gamma, n)$ reactions on $\mathrm{Pb}$ and $\mathrm{Bi}$. Also the importance of experimental data is strongly emphasized by these findings. Because of the magicity or near-magicity of the $\mathrm{Pb}$ and $\mathrm{Bi}$ isotopes, individual resonances determine the cross sections and the Hauser-Feshbach theory is not applicable $[32,33]$. From discrepancies between resonance and activation measurements [34,35] and from theoretical considerations [36], it has been previously found that even a small direct capture component contributes to neutron capture on $\mathrm{Pb}$ $[32,36]$. Resonant and direct capture contributions are difficult to handle in theoretical models and experiments prove to be indispensable.

We thank E.P. Knaetsch, D. Roller and W. Seith for their help and support during the neutron irradiations at the Karlsruhe Van de Graaff accelerator. This work was supported by the Swiss National Science Foundation Grants 2024-067428.01 and 2000-105328.

\section{References}

1. B. Allen, J. Gibbons, R. Macklin, Adv. Nucl. Phys. 4, 205 (1971).

2. Z. Bao, Käppeler, At. Data Nucl. Data Tables 36, 411 (1987).

3. H. Beer, F. Voss, R. Winters, ApJ Suppl. 80, 403 (1992).

4. Z. Bao, H. Beer, F. Käppeler, F. Voss, K. Wisshak, T. Rauscher, At. Data Nucl. Data Tables 76, 70 (2000).

5. T. Rauscher, F.-K. Thielemann, At. Data Nucl. Data Tables 75, 1 (2000).

6. I. Dillmann, M. Heil, F. Käppeler, R. Plag, T. Rauscher, F.-K. Thielemann, Proc. 12th International Symposium on Capture Gamma-Ray Spectroscopy and Related Topics (CGS 12), Notre Dame, IN/USA, AIP Conf. Proc. 819, 123 (2006).

7. E. Anders, N. Grevesse, Geochim. Cosmochim. Acta 53, 197 (1989).

8. K. Rosman, P. Taylor, Pure Appl. Chem. 70, 217 (1998).

9. M. Rayet, N. Prantzos, M. Arnould, A\&A 227, 271 (1990).

10. M. Rayet, M. Arnould, M. Hashimoto, N. Prantzos, K. Nomoto, A\&A 298, 517 (1995)
11. C. Fröhlich, G. Martinez-Pinedo, M. Liebendörfer, F.-K. Thielemann, E. Bravo, W.R. Hix, K. Langanke, N.T. Zinner, Phys. Rev. Lett. 96, 142502 (2006).

12. H. Schatz, A. Aprahamian, J. Görres, M.C. Wiescher, T. Rauscher, J. Rembges, F.-K. Thielemann, B. Pfeiffer, P. Möller, H. Herndl et al., Phys. Rep. 294, 167 (1998).

13. W. Rapp, J. Görres, M. Wiescher, H. Schatz, F. Käppeler, ApJ 653, 474 (2006).

14. I. Dillmann, Ph.D. thesis, University of Basel, 2006.

15. W. Hauser, H. Feshbach, Phys. Rev. 87, 366 (1952).

16. W. Ratynski, F. Käppeler, Phys. Rev. C 37, 595 (1988).

17. S. Dababneh, N. Patronis, P.A. Assimakopoulos, J. Görres, M. Heil, F. Käppeler, D. Karamanis, S. O’Brien, R. Reifarth, Nucl. Instrum. Meth. A 517, 230 (2004).

18. A.R. Farhan, B. Singh, Nucl. Data Sheets 86, 785 (1999).

19. H. Sievers, Nucl. Data Sheets 62, 271 (1991).

20. D. de Frenne, E. Jacobs, Nucl. Data Sheets 93, 447 (2001).

21. T. Tamura, Nucl. Data Sheets 90, 107 (2000).

22. Yu.V. Sergeenkov, Yu.L. Khazov, T.W. Burrows, M.R. Bhat, Nucl. Data Sheets 72, 487 (1994).

23. R. Shaheen, Nucl. Data Sheets 75, 491 (1995).

24. Yu.V. Sergeenkov and V.M. Sigalov, Nucl. Data Sheets 49, 639 (1986).

25. R.G. Helmer, Nucl. Data Sheets 103, 565 (2004).

26. M.S. Basunia, Nucl. Data Sheets 102, 719 (2004).

27. Z. Chunmei, Nucl. Data Sheets 95, 59 (2002).

28. I. Dillmann, M. Heil, F. Käppeler, T. Rauscher, F.-K. Thielemann, Phys. Rev. C 73, 015803 (2006).

29. C. Vockenhuber, I. Dillmann, M. Heil, F. Käppeler, N. Winckler, W. Kutschera, A. Wallner, M. Bichler, S. Dababneh, S. Bisterzo, R. Gallino, Phys. Rev. C 75, 015804 (2006).

30. W. Rapp, Report FZKA 6956, Forschungszentrum Karlsruhe (2004).

31. Online: http://download.nucastro.org/astro/reaclib

32. A.M. Lane, J.E. Lynn, Nucl. Phys. 17, 586 (1960).

33. T. Rauscher, F.-K. Thielemann, K.-L. Kratz, Phys. Rev. C 56, 1613 (1997).

34. R.L. Macklin, J. Halperin, R.R. Winters, ApJ 217, 222 (1977).

35. H. Beer, F. Corvi, P. Mutti, ApJ 474, 843 (1997).

36. T. Rauscher, R. Bieber, H. Oberhummer, K.-L. Kratz, J. Dobaczewski, P. Möller, M.M. Sharma, Phys. Rev. C 57, 2031 (1998). 